\title{
UN ACERCAMIENTO FENOMENOLÓGICO A LA CUESTIÓN DE LA AGENCIA TÉCNICA EN MERLEAU-PONTY
}

\author{
LETICIA BASSO MONTEVERDE \\ Universidad Nacional de Mar del Plata - CONICET, Argentina
}

\begin{abstract}
RESUMEN: Espacio, cuerpo y hábito delimitan en este trabajo el entorno en el cual se emplaza el tema de la agencia técnica, en tanto una modalidad de la praxis que ejerce el sujeto en el mundo. En Phénoménologie de la perception Merleau-Ponty hace un análisis que ayuda a rastrear cómo se conforma su sentido en relación a la situación a la que se circunscribe el comportamiento del sujeto con los útiles en la experiencia. Una reconstrucción de estas prácticas requerirá exhibir los mecanismos vitales que plasman una versión efectiva del horizonte de posibilidades del útil. Hay algo que escapa a la lectura teórica, que tiene que ver con la fuerza y la intensidad de las percepciones inherentes a la conducta del sujeto «encarnado». Este plus de sentido que excede el rango objetivo contribuye, sin más, a la constitución elemental de la práctica de una manera que sólo una exploración directa del acto y su sentir nos provee. De este modo, se puede abordar la complejidad y limitaciones de la experiencia que aparecen con una lectura inmanente de su sentido.
\end{abstract}

PALABRAS CLAVE: inmanencia; mundo vivido; útiles; práctica; sentido.

\section{A phenomenological approach to the issue of technical agency in Merleau-Ponty}

ABSTRACT: Space, body and habit delimit in this work the environment in which the issue of technical agency is located, as a modality of praxis that the subject exercises in the world. In Phénoménologie de la perception Merleau-Ponty makes an analysis that helps to trace how its meaning conforms to the situation in which the behavior of the subject with tools in experience is circumscribed. A reconstruction of these practices will require exhibiting the vital mechanisms that shape an effective version of the tool's horizon of possibilities. There is something that escapes theoretical reading, which has to do with the strength and intensity of the perceptions inherent in the «incarnate» subject's behavior. This plus of meaning that exceeds the objective range contributes, without more, to the elemental constitution of the practice in a way that only a direct exploration of the act and its feeling provides us. In this way, the complexity and limitations of the experience that appear with an immanent reading of its meaning can be addressed.

KEY WORDS: immanence; lived world; tools; practice; meaning.

\section{INTRODUCCIÓN}

Espacio, cuerpo y hábito son categorías que delimitan el entorno en el cual se emplaza el tema de la agencia técnica, en tanto una modalidad de la praxis que ejerce el sujeto en el mundo. En este caso particular, restringimos el concepto de agencia técnica al ámbito de posibilidades que se gestan en la relación del sujeto con los útiles, dicho ámbito ya muestra la hibridación ontológica de lo natural y lo cultural. Por ende, la praxis es el efecto de un sujeto que habita y produce sentido en el marco de un mundo tecnificado, en el cual tanto su accionar como su forma 
de comprenderse en ese proceso implican una experiencia holística e integrada del trato con útiles.

Para explorar adecuadamente el tema y orientar la mirada hacia una forma específica de comprender su génesis, recurrimos a Merleau-Ponty quien aporta — principalmente en su libro Phénoménologie de la perception (1945)— un análisis descriptivo de la percepción y uso de útiles que ayuda a rastrear: (1) cómo se conforma su sentido en relación a la situación en la que se circunscribe el comportamiento del sujeto en la experiencia. El concepto de sentido lo interpretamos desde la tradición fenomenológico-hermenéutica, la cual propone establecer una lectura preteórica acerca del trasfondo de comprensión que regula nuestro modo de ser en el mundo. De esta manera, la intención del trabajo es acentuar: (1.a) cómo en el marco de esta modalidad de la praxis intervienen los útiles y (1.b) de qué manera nuestro comportamiento ya está predelineado por el efecto de esas prácticas instituidas.

Ahora bien, al reconstruir los factores que determinan las prácticas originarias al trato con útiles es preciso exhibir la lógica particular de estos actos in situ ${ }^{1}$. Para ello, el filósofo nos aporta una visión antidualista en la cual se puede apreciar con claridad cómo el propio sujeto es un producto de ese mundo de relaciones, y de qué manera la corporalidad y el espacio se definen por esas prácticas. Esto se debe a que las características que podemos rescatar para formular la estructura y la dinámica de esta clase de experiencias provienen, indiscutiblemente, del estudio fáctico de situaciones concretas.

Bajo esta óptica, se puede efectuar un seguimiento de los mecanismos vitales que plasman una versión efectiva del horizonte de posibilidades de dicho útil. Pues bien, consideramos que hay algo que escapa a la lectura teórica del trato con el útil, que tiene que ver con la fuerza y la intensidad de las percepciones inherentes a la conducta del sujeto «encarnado». Este plus de sentido, que excede el rango de objetivación, contribuye a la constitución elemental de la práctica, de una manera que sólo una exploración directa del acto y su sentir nos provee.

Merleau-Ponty nos dice: «el sentir siempre involucra al cuerpo. El problema estriba en comprender estas relaciones singulares que se tejen en las partes del paisaje entre sí o entre éste y yo como sujeto encarnado [...] El sentir es esta comunicación vital con el mundo que nos lo hace presente como lugar familiar de nuestra vida. $»^{2} \mathrm{Al}$ respecto, el filósofo se dedica precisamente a examinar estos rasgos vitales con la finalidad de descubrir cómo afectan al sujeto y qué producen. Intentaremos direccionar estos interrogantes a la cuestión del trato con el útil, tal como lo presentamos en esta introducción.

1 Merleau-Ponty sostiene en este libro que «...el "algo" perceptivo está siempre en el contexto de algo más; siempre forma parte de un "campo" [...] La estructura de la percepción efectiva es la única que puede enseñarnos lo que sea percibir. La impresión pura no sólo es, pues, imposible de hallar, sino también imperceptible [...] un dato perceptivo aislado es inconcebible». (Merleau-Ponty, M., Phénoménologie de la perception, Gallimard, Paris, 1945, p. 10. /Trad. Fenomenología de la percepción, Planeta-Agostini, Barcelona, 1993, p. 26.) A partir de ahora, las citas de este libro y su traducción se harán abreviando el título con la sigla $P h p$ y a continuación se indicará la paginación del original y la traducción seleccionada.

2 Merleau-Ponty, $P h p$, p. 64/p. 73. 


\section{El trato CON El Útil COMO Un MOdo DE SER-EN-EL-MUNDO}

A la hora de precisar qué es un útil no debemos incurrir en el error de elaborar una definición sustancialista que establezca la naturaleza de estas entidades por medio de cualidades objetivas. En realidad debemos responder a la pregunta ¿cómo se emplea un útil? Allí encontraremos la modalidad apropiada de acceder a estas entidades. Esta lectura ya la introduce Heidegger en Sein und Zeit (1927) cuando aclara que el rasgo determinante de estos entes lo da su uso, ya que en su «para-qué» (Wozu) reside el sentido acerca del cual se articula una forma de «seren-el-mundo» —en este caso, la de la Zuhandenheit (el estar-a-la-mano del útil).

El mismo Husserl nos dice al respecto en el segundo libro de Ideen zu einen reinen Phänomenologie und phänomenologischen Philosophie: "Conocer una cosa quiere por ende decir: saber por experiencia cómo se conduce al presionarla y golpearla, al doblarla y romperla, al calentarla y enfriarla, esto es, cómo se comporta en el nexo de sus causalidades, en qué estados entra, cómo al atravesarlos sigue siendo la misma $»^{3}$. De esta manera, comprender el sentido y la función del útil involucra adentrarse en las prácticas que llevamos acabo con este tipo de entidades. Pues, ellas nos aportan otro modo de «ser-en-el-mundo», uno que se configura a partir de nuestro hacer y que, como tal, asume otra significación del entorno basada en nuestras habilidades motrices.

$\mathrm{Al}$ respecto, Merleau-Ponty establece que el asunto de «ser-en-el-mundo» o «ser del mundo» (être au monde) no debe reducirse al resultado de un proceso de significación subjetiva ni del influjo de causalidades externas. Esta modalidad de la práctica no se resume a un mecanismo dual de nuestro hacer. El mundo tiene opacidades que sobrepasan la actividad sintética de la conciencia y, por esto, «es» antes de que el sujeto asuma la labor de comprender su sentido. Basta señalar que, al ensayar una descripción del uso de útiles aparecen elementos que rebasan la intencionalidad presente del que los inventa o utiliza. Con esto queremos decir, que esta modalidad de la praxis no se transparenta a la conciencia que ejerce el acto presente.

El uso de útiles depende de un trasfondo cultural que previamente lo condiciona. A su vez, este trasfondo se activa cuando el sujeto ejecuta el acto en cuestión ${ }^{4}$. Como el uso suele contextualizarse en una experiencia pre-teórica, no apunta a

3 HusserL, E., Ideen zu einen reinen Phänomenologie und phänomenologischen Philosophie. Zweites Buch: Phänomenologische Untersuchungen zur Konstitution, Martinus Nijhoff, The Hague, 1952, p. 45. [Trad. Ideas relativas a una fenomenología pura y una filosofía fenomenológica. Libro II: Investigaciones fenomenológicas sobre la constitución, FCE, México, 2005, p. 75.]

4 Nicola Liberati retoma los análisis de Verbeek en What Things do: philosophical reflections on technology, agency and desing (2005) y comenta que «...es necesario considerar que el uso de tecnología no es neutral, sino que estructura la percepción e incluso a la subjetividad» (Cf. Liberati, N., "Leib and Technologies: Relations and co-Fundation» en Investigaciones fenomenológicas, $\mathrm{N}^{\circ} 11,2014$, pp. 165-184- traducción propia) Entonces, el uso particular que hacemos de un útil determina nuestra forma de comprender el entorno y, a la vez, este uso no suele ser azaroso o circunstancial porque está marcado por una serie de decisiones que llevaron a su institución. El tema es que el sujeto que la lleva a cabo dicha acción no es necesariamente consciente de ellas. 
una toma de posición deliberada frente al trasfondo determinante. Más bien, éste se resuelve en una actitud semiconsciente por el cruce de intereses presupuestos y habilidades adquiridas. La agencia técnica, en este sentido, se despliega en una serie de capas o niveles de la conciencia. Algunos de ellos se efectúan bajo un estado consciente o deliberativo y otros operan implícitamente a medida que el sujeto involucrado en la práctica se compenetra con la actividad en proceso.

Esta serie de condiciones sedimentadas, que se alojan en el sujeto y lo llevan a responder de cierta forma, son el efecto del influjo de la tradición y de las construcciones sociales, simbólicas e ideológicas que dominan la «versión del mundo» que allí se activa. Merleau-Ponty brinda, incluso, ejemplos del uso de útiles que permiten visibilizar cómo estas entidades pasan a formar parte de nuestra corporalidad en el momento en el que comportan una extensión de nuestra capacidad sensorio-motora. Hay ejemplos puntuales del filósofo, como el uso del bastón para el ciego, que ilustran cómo los útiles puede aportarnos un modo de expresión y orientación en el entorno - más adelante retomaremos esta cuestión.

En suma, la percepción y el uso de útiles dependen de una escena que, por un lado, tiene que ver con el cumplimiento de una intención determinada frente a una tendencia actual del sujeto pero, por otro lado, conllevan motivos latentes que encaminan la acción y prefiguran el campo fenomenal en el que se insertan ${ }^{5}$. Por ende, con el uso cotidiano de entidades de esta índole la acción se modela a partir de factores determinados que corresponden a la experiencia singular que se presenta y factores indeterminados $\mathrm{u}$ ocultos que anticipan $\mathrm{y}$, de esta forma, modelan el acceso y trato que se genera. Por consiguiente, en la teoría merleaupontyana la percepción y el uso de útiles se desarrollan en el contacto directo, primigenio y concreto. Dicho contacto se manifiesta de manera previa a toda reflexión, aunque en un marco de sentido en el cual se maneja el sujeto como totalidad significante e indivisa. La totalidad es vivida y habitada bajo una forma de uso que, a simple vista, parece ya constituida.

En otras palabras, el sujeto mantiene una actitud intuitiva en el trato cotidiano que se caracteriza por cierta familiaridad de orden práctico. No obstante, esta familiaridad aporta de modo encubierto una serie de significaciones instituidas que articulan la forma y el contenido de las experiencias. Así: «Es esencial a todo aparecer perceptivo que el objeto se dé como estando ya ahí antes de que yo lo descubra, y que se dé además como abierto a mi exploración con la posibilidad de desplegar

5 Thomas Buhrman y Ezequiel Di Paolo tratan esta tensión entre una consideración del sentido de la agencia desde una perspectiva internalista o una reticular. La primera se concentra en una lectura del agente como autor o iniciador de sus propias acciones que levemente tienden a modificarse por eventos externos o circunstancias objetivas y la segunda considera al sujeto como un yo corporizado que a partir de esquemas sensorio-motores atiende a las relaciones significativas que se plasman en el mundo. Esta última lectura aporta una visión más compleja de la constitución de la estructura de la percepción y la agencia en sí misma. Consideramos que la propuesta de Merleau-Ponty podría encuadrarse en esta segunda perspectiva. (Cf. Buhrman T.; Di PaOlo, E., «The sense of agency. A phenomenological consequence of enacting sensorimotor schemes» en Phenomenology and The Cognitive Sciences Journal, noviembre, DOI 10.1007/s11097-015-9446-7, 2015.) 
más aspectos que los que me son actualmente presentados y proponerme comportamientos distintos de los que anticipé al verlo» ${ }^{6}$.

Dicho brevemente, en el trato con el útil el sujeto se comporta de manera activa y pasiva. Esto se debe a que este estilo de prácticas tiene un costado productivo, puesto que una de las formas de entenderlo es como medio generado para acceder a un objetivo pergeñado. Mas aún, el manejo que de éste realizamos se supedita a cierta destreza que excede generalmente al acto presente. Por lo cual, el acto procede y se efectúa bajo la forma de una capacidad ya incorporada, esto es lo que suele entenderse por el concepto de hábito.

En consecuencia, la práctica se realiza de forma a-temática como habilidad que traemos de otras experiencias o de la formación que se afianza en generaciones pasadas. Para ilustrarlo con un simple ejemplo: «...no habría aparecido nunca la silla como silla si yo no tuviera en mi repertorio de posibilidades motrices el comportamiento de sentarme» ${ }^{7}$.

Como resultado, por más que el sujeto se restrinja a un acto puntual que considere evidente por sí mismo, el trasfondo en el que se ejecuta e instaura el útil se ciñe a una dimensión de sentido potencial, abierta y unitaria que permanece oculta al sujeto pero posibilita todo direccionamiento y atención perceptivas. Entonces, la trama compleja de relaciones significativas que componen y condicionan el uso de este tipo de entidad se realiza en el micro-contexto del mundo experimentado, aunque es viable gracias al macro-contexto intersubjetivo que la respalda. Con todo, es central el papel del sujeto porque más allá de que se encuentre determinado por el horizonte de sentido en el cual se encuentra, él es «...la fuente absoluta que retoma, reanuda y sostiene esos antecedentes del medio físico y social» ${ }^{8}$.

Por este motivo, la fenomenología tiene un doble desafío: por un lado, debe captar la evolución de la práctica de la forma más neutral, es decir, sin intervenir en cómo efectivamente acontece. Ella debe resguardarse de tergiversar la originalidad de la práctica y la inmediatez con la que se desarrolla. Por el otro lado, en esta relación del sujeto con la sensibilidad y la motricidad primitivas que caracterizan al uso del útil, se imponen implícitamente otro tipo de constricciones que contradicen el estado puro pretendido porque, de hecho, no existe tal cosa. Hay factores del orden «tético» que afectan a la práctica de forma previa y que, incluso, la obligan a reconfigurarse en medio de la experiencia. Más adelante explicaremos esto.

En este sentido, el sujeto no es una hoja en blanco; no inicia su experiencia desde un grado cero. Como ser histórico, fáctico y cultural está filtrado por significaciones y modalidades de la praxis que marcan su experiencia. Así pues, expresa el filósofo francés: «En el hombre todo es fabricado y todo es natural [...]»9. De manera que, a la hora de mostrar cómo se constituye el sentido en el sujeto, la fenomenología tropieza con estas contradicciones de su objeto de estudio. Con esto, se adelanta que el tipo de concepción a la que se intenta arribar no presenta al sentido como el resultado, de antemano consumado, a partir de la cual se emplea un útil.

6 García, E., Maurice Merleau-Ponty. Filosofía, corporalidad y percepción, Editorial Rhesis, Buenos Aires, 2012, p. 92.

7 Ibidem.

8 Cf. Merleau-Ponty, $P h p$, p. III/ pp. 8-9

9 Merleau-Ponty, Php, p. 221/ p. 206. 
Por el contrario, el sentido se conforma mediante la actividad cotidiana del sujeto; ni es independiente de la experiencia ni lo condiciona de manera exclusiva.

Sin duda alguna, esta perspectiva fenomenológica encauza la lectura a una definición de la práctica determinada por la articulación, la fundación y la transformación del mundo en donde el sujeto «es» y que, a su vez, hereda. Esto sucede porque se constituye en relación a un entramado de vivencias y acontecimientos que marcan su existencia. La tarea de la fenomenología es la de explicitar cómo se produce este entramado que condiciona al sujeto y a partir del cual instituye el mundo en donde actúa. Pues bien, en el «mundo de la vida» (Lebenswelt) que está en juego se encuentran las pautas precisas para toda apropiación, re-elaboración y actualización de sus contenidos.

A continuación profundizaremos en las claves fenomenológicas de espacio, cuerpo y hábito con el interés de exponer mejor cómo se constituyen la agencia técnica en la modalidad del trato con útiles.

\section{La PREgUNTA POR LA ESPACIALIDAD DE LA PRÁCticA EN TORNO AL ÚtIL}

Esta idea de «la versión del mundo» —que el filósofo francés rescata bajo el concepto de mundo vivido (monde vécu $)^{10}$ — se encuadra en una manera específica de comprender la espacialidad, dado que el fenómeno de la percepción se despliega en un ámbito en donde el sujeto y el útil convergen. A nuestro juicio, la espacialidad no alude estrictamente a un mundo relativo y privado del sujeto ni a un mundo exterior y trascendente a él, sino que se constituye por el cruce de ambos ámbitos. De este modo, el espacio no se deduce de la revisión introspectiva de una postura intelectualista ni se avista meramente bajo la modalidad del cálculo que lleva a cabo una actitud empirista.

Pareciera que ningún hecho o propósito corta los hilos intencionales de la experiencia del «mundo vivido» para dar cuenta de lo exterior. Pues bien, no puede traspasarse esta forma de percibir el mundo para acceder a las cosas y el espacio como una especie de realidad en $s i^{11}$. Sin embargo, consideramos que hay momentos en los que la acción demanda un reposicionamiento y contrastación de las condiciones externas al entramado de sentido del «mundo vivido» para conducir las tendencias del sujeto hacia motivaciones precisas. Pues, ciertamente, la experiencia

10 La noción de mundo vivido consiste en una deriva de las fórmulas de «vivir en el mundo» (In-der-Welt-Leben) y "ser en el mundo» (In-der-Welt-Sein). A través de esta noción Merleau-Ponty condensa y refuerza el sentido fáctico, histórico y existencial de la experiencia del sujeto, que se encuentra plasmado en buena parte de los trabajos de sus predecesores -Husserl y Heidegger. El concepto de mundo vivido, que Merleau-Ponty presenta de forma contundente en su obra capital, es la profundización de la crítica fenomenológica a la postura clásica de la filosofía moderna acerca de la relación entre el hombre y el mundo. El filósofo francés quiere dejar atrás la perspectiva dualista que dispone enfrentados a sujeto-objeto, conciencia-cuerpo, yo-otro.

11 Para un análisis acerca de la imposibilidad de percibir lo real en sí mismo en el pensamiento de Merleau-Ponty, véase el apartado «External Reality» de Pietersma, H., Phenomenological Epistemology, Oxford University Press, New York-Oxford, 2000, pp. 141 y ss. 
del sujeto debe habilitar y restituir una serie de habilidades que convengan para la realización del acto. De esta manera, si se tiene en cuenta la influencia de Husserl, la actitud teórica conllevaría un instinto objetivante que motiva al sujeto a alcanzar el cumplimiento de esta intención. Para esto se hace imprescindible producir las habilidades y sus objetos correlativos ${ }^{12}$. Como señala Merleau-Ponty:

Todas esas operaciones exigen un mismo poder de trazar en el mundo dado unas fronteras, unas direcciones, establecer unas líneas de fuerza, preparar unas perspectivas, en una palabra, organizar el mundo dado según los proyectos del momento, construir en el marco geográfico un medio contextual de comportamiento, un sistema de significaciones que expresen al exterior la actividad interna del sujeto ${ }^{13}$.

Con todo, la fenomenología escapa al cruce entre el plano vivido y la realidad externa. Por ejemplo, refiriéndose a la imposibilidad de una adecuación de las percepciones al espacio exterior, Serrano de Haro señala en su libro La precisión del сиегро:

...la interiorización más plena de la dinámica de movimientos corporales no alcanzará nunca a transformar, a trasmutar la percepción externa del espacio; no transformará la distancia intuitiva que veo, en vivencia inmanente que vivo [...] o, a la inversa, no transfigurará la intimidad de mi conciencia en la solidez y extensión de la cosa o del útil. Por tanto, la habituación tampoco acertará a desembocar en un ámbito neutro e indefinido que no sea ni experiencia vivida ni correlato objetivo de la experiencia [...] Dicho en otras palabras, el curso del acto y el cumplimiento del acto descansan sobre el imperio infrangible de la percepción sensible, que trae a intuición la situación espacial y hace patente un panorama concreto en relación con mi propio cuerpo. Ningún hábito puede suspenderla, ninguno agotarla ${ }^{14}$.

De este modo, la experiencia, la situación espacial e incluso la captación y uso del útil responden a la forma de disponer, atender, reconocer el mundo en donde vivimos. No hay en la experiencia un estado en el cual las cosas se presenten más allá de la forma de entenderlas o significarlas. Cada una de las percepciones, cada valoración del entorno e, incluso, en cada ocasión que nos atenemos a elaborar un juicio que ostente validez objetiva, nos apoyamos en el mundo que habitamos y nos habita. Esta postura es poco flexible y entendemos que puede discutirse. Pero como toda percepción alude a partes de objetos bajo escorzos o figuras puntuales, la sumatoria de actos que emprendamos con tal entidad no dará nunca una visión acabada y, por ende, es en cierta forma infinito el horizonte de posibilidades de experimentación de esa cosa. Incluso, si tenemos en cuenta la idea merleaupontyana de que los escorzos no se presentan solos, sino en función de apariciones ya preconcebidas de objetos que vienen dadas por nuestro repertorio de significaciones, siempre habrá un fondo indeterminado a la espera de explicitar otro tipo de vínculo con lo dado. En este sentido es que no salimos del mundo vivido, porque

12 Véanse, García, o.c., p. 62.

13 Merlau-Ponty, Php, p. 130/p. 129. El subrayado es nuestro.

14 Serrano de Haro, A., La precisión del cuerpo. Análisis filosófico de la puntería, Trotta, Madrid, 2007, pp. 59-60. 
siempre hay una forma nueva de experimentar la cosa que se configura ${ }^{15}$. Como marca Merleau-Ponty en su texto: «...el espacio y el tiempo que yo habito tienen siempre, por una parte y otra, unos horizontes indeterminados que encierran otros puntos de vista» ${ }^{16}$.

Aunque Merleau-Ponty explica en su libro que ese fondo indeterminado es la base para la proyección del sujeto, pues despierta en el cuerpo que vive una situación concreta, un «movimiento virtual» donde la parte del cuerpo interrogada sale del anonimato ${ }^{17}$. Razón por la cual el fondo correspondiente al «movimiento virtual» que se dispone en potencia, empuja al sujeto a construir las condiciones exteriores para llevar a cabo el acto. Pues, lo impulsa a producir las cinestesias para que «... lo que no existe naturalmente pueda tomar un semblante de existencia» ${ }^{18}$. La cuestión a discutir es si esta producción es inmanente al espacio del mundo vivido o si tiene en cuenta al espacio exterior en el que las cosas aparecen como objetos en sí, en vista a incorporarse a la red de intenciones del sujeto. Pues, en definitiva la cosa es una y las causalidades de la naturaleza, a nuestro juicio, no dejan de afectar la experiencia.

Al respecto Merleau-Ponty ilustra el caso: «Para que una llave, por ejemplo, aparezca como llave en mi experiencia táctil, se necesita una especie de amplitud del tacto, un campo táctil en el que las impresiones locales puedan integrarse en una configuración» ${ }^{19}$. De esta manera, el espacio se proyecta como el escenario vital en el cual el sentido de la práctica se ajusta a medida que las anticipaciones del sujeto y las daciones del objeto adquieren una organización precisa. Hay que ver si este ajuste atañe al interior de la práctica o si es una manera de incorporar algo que excede lo vivido. «El movimiento virtual abre al interior del mundo pleno en el que se desarrollaba el movimiento concreto una zona de reflexión y de subjetividad, superpone al espacio físico un espacio virtual o humano [...] que desarrolla él mismo su fondo [...] al reservar delante de sí un espacio libre para la creación o modificación de lo dado ${ }^{20}$. Todavía no queda claro si ese espacio es una ampliación del mundo vivido o una conexión con el espacio exterior. Sí parece que tal producción genera una modificación de la actitud del sujeto en el trato con el útil, ya que precisa salir del anonimato para crear las condiciones necesarias a fin de continuar con la práctica. Ahora bien, habrá que ver si la actitud teórica se asocia a una lectura del espacio exterior. Retomaremos la explicación del proceso para ver a dónde nos conduce. Para ello nos introduciremos en el tema del cuerpo.

15 Serrano de Haro retoma a Husserl y comenta que «...este es un proceso de suyo infinito; que puede proseguir siempre, sin que con ello devenga una eterna repetición de lo mismo, sino en todo caso variaciones significativas sobre lo mismo. En la plenitud del detalle y de la claridad, el proceso está indefinidamente pendiente de cerrarse; a cada paso trae consigo nuevas anticipaciones y previsiones, y retrospectivamente pide constantes revisiones y reactualizaciones de los apareceres previos [...] de suerte que no cabe el acabamiento de la captación.» (Ibidem, pp. 87-88)

16 Merleau-PonTy, Php, p. 164/p. 157.

17 Cf. Ibidem, p. 126/p. 125.

18 Cf. Ibidem, p. 129/p. 128.

19 Ibidem, p. 126/p. 126.

20 Cf. Ibidem, p. 129/p. 128. Subrayamos los términos «espacio físico» y «delante de sí» porque creemos que acentúan la idea de un espacio exterior. 


\section{El PAPEL DEL CUERPo EN El trato CON los Útiles}

Según Merleau-Ponty el propio cuerpo no es un objeto más a experimentar en el mundo porque «...se me revela como postura en vistas a una cierta tarea actual o posible» ${ }^{21}$. Por ende, el cuerpo se estructura a partir de la dinámica de la práctica en cuestión, y ofrece resistencia y permanencia frente a la variación de componentes que en torno a él aparecen ${ }^{22}$. Esta constancia y tenacidad la obtiene del esquema corpóreo por medio del cual experimenta o crea las cinestesias del momento en relación a su constitución entera, además de la serie de habilidades que posee y que pueden servir para modelar su entorno. Así, se hace preciso que el sujeto «... invierta la relación natural del cuerpo y de la circunstancia inmediata y que una productividad humana se abra camino a través de la espesura del ser» ${ }^{23}$.

Entonces, el espacio se habita como presencia viva e irrecusable de lo carnal. El cuerpo es el lugar en donde convergen estas tensiones pues: «El "aquí" inalienable del espesor carnal [...] es también el aquí itinerante o ambulante que define los ejes del panorama perceptivo y los desplaza consigo ${ }^{24}$. En otras palabras, el espacio experimentado por el sujeto es el espacio corpóreo en sus distintas configuraciones perceptivas y motrices. Al respecto Merleau-Ponty indica: «...las determinaciones espaciales de lo percibido, e incluso la presencia o la ausencia de una percepción, no son efectos de la situación efectiva al exterior del organismo, si no que representan la manera como éste va al encuentro de unas estimulaciones y cómo se remite a las mismas» ${ }^{25}$.

Por lo tanto, en el pensamiento de Merleau-Ponty encontramos efectivamente una relación entre la idea de un espacio exterior y el espacio corpóreo ${ }^{26}$. Por el hecho de que, todo acto perceptivo articula la tensión figura-fondo atendiendo a la orientación del propio cuerpo como tercer elemento en el que se emplaza la encrucijada de lo interno y lo externo. Ya señala el filósofo al inicio de la segunda parte de su texto que: «La percepción exterior y la percepción del propio cuerpo varían conjuntamente porque son las dos caras de un mismo acto. [Así...] cierta forma de experiencia externa implica y comporta una cierta consciencia del propio cuerpo. [Y...] Toda percepción de mi cuerpo se explicita en el lenguaje de toda percepción exterior ${ }^{27}$.

La fenomenología, de la mano de Husserl y Merleau-Ponty, contribuye en gran medida a la investigación de estas cuestiones al focalizar la experiencia sensorial en el cuerpo. Cuando tematiza la corporalidad abre un ámbito de significación de lo espacial en el cual se desenvuelven las series funcionales de dependencias

21 Cf. Ibidem, p. 117/p. 117.

22 A propósito dice Esteban García: «A diferencia de todo objeto percibido, hay una permanencia absoluta del cuerpo en el campo perceptivo que determina a la vez una limitación absoluta de la posibilidad de variar de perspectiva respecto suyo.» (GARCíA, o.c., $\mathrm{p}, 125)$

23 Merleau-Ponty, Php, p. 130/p. 129.

24 Serrano de Haro, o.c., p. 85

25 Merleau-Ponty, Php, p. 89/p. 94.

26 Ibidem, p. 118/p. 118 y ss.

27 Ibidem, p. 239/p. 222. 
y habilidades que se sintetizan en circunstancias «reales» y aluden a la acción, el sujeto y el objeto involucrados. Merleau-Ponty dice que «...no puede comprenderse la función del cuerpo viviente más que llevándola uno a cabo y en la medida en que uno sea un cuerpo que se eleva hacia el mundo ${ }^{28}$. Esta es la visión merleaupontyana del sujeto encarnado, un sujeto que es en y del mundo gracias a la corporalidad ${ }^{29}$. El cuerpo es el medio desde el cual irradia y se imprime un cúmulo de cinestesias que hace personal y única a la experiencia, y con ella, al manejo del útil en cuestión. De hecho, el uso de los útiles se da a través del cuerpo, a tal punto que el útil empleado puede llegar a incorporarse a la estructura como si fuera una extensión protésica o una parte encarnada. Al respecto existe todo un ámbito de discusión sobre si los útiles pueden modificar el propio cuerpo. Es decir, si los útiles deben considerarse como una mera extensión a emplear o si pueden tenerse por una parte del cuerpo vivido que a largo del uso modela, incluso, la constitución corporal ${ }^{30}$. En el ámbito fenomenológico Serrano de Haro lo reconstruye del siguiente modo:

...el perfeccionamiento exhaustivo de un orden muy preciso de movimientos [...] termina transformando determinados actos [...] en una casi prolongación de la movilidad corporal. La repetición y corrección constantes durante largos períodos de tiempo logran una interiorización plena de la práctica, que alcanza unitariamente a todas las dimensiones: a los esquemas de la percepción, al control anímico y motriz, a la captación y fortalecimiento del cuerpo, etc. El grado de familiaridad llega a ser tal, que hasta los útiles empleados se tornan una suerte de prótesis corporal ${ }^{31}$.

Empero, retomando el eje de nuestra lectura sostenemos que es en el cuerpo y por medio del cuerpo que acontecen sensaciones referentes específicamente al trato del útil allí consolidado. Estas sensaciones son la base originaria de toda estructura perceptiva. De igual manera, no debemos olvidar que la motricidad del cuerpo es condición de posibilidad para todo estado representativo de dicho espacio y objeto. Esto es así porque la motricidad ya es dadora de sentido en un plano elemental y primigenio. De ahí que, en un sentido cronológico debamos afirmar que antes de la predicación y de toda forma de pensamiento poseemos un sentido a través del cuerpo. Pero, a su vez, no podemos olvidar que como seres históricos y culturales experimentamos el mundo, de entrada, con un bagaje conceptual que determina nuestras maneras de acceder al mundo, incluso en la serie de movimientos que creemos ejecutar automáticamente.

Ahora bien, en cuanto al uso de útiles hay que agregar que el sujeto se encuentra en la experiencia totalmente abocado a este sentir y abstraído, en principio, de las causas y los factores que posibilitan el acto. Dijimos que el sujeto está encarnado; es su cuerpo porque vive, siente y piensa desde él. El cuerpo juega un papel esencial ya que de acuerdo a su localización y orientación emprende un vínculo con el útil.

28 Ibidem, p. 90/p. 94.

29 En este sentido, la concepción merleaupontyana de mundo vivido dista de presentar al sujeto como una conciencia pensante que además mantiene en la experiencia sensible una conexión con su cuerpo, puesto que en realidad la corporalidad es lo que prima. Somos sujetos corporales con la capacidad de pensar, no sujetos con cuerpo.

30 Sobre esta discusión véase el artículo de Liberati, o.c., p. 176 y ss.

31 Serrano de Haro, o.c., p. 58. 
De esta forma, hay una referencialidad al cuerpo que es central para la constitución de estas entidades. Por ejemplo, el filósofo francés señala: «El bastón del ciego ha dejado de ser un objeto para él, ya no se percibe por sí mismo, su extremidad se ha transformado en zona sensible, aumenta la amplitud y el radio de acción del tacto...» ${ }^{32}$ En suma, el uso de útiles está subordinado a la corporalidad, a cómo el cuerpo interactúa con éstos. Esta manera de presentar el manejo de los útiles está fuertemente influenciada por la elaboración heideggeriana. Merleau-Ponty también considera que en el trato con el útil se desarrolla un tipo de significación pre-reflexiva en la que se activan de manera inmediata una serie de habilidades incorporadas y sus consecuentes aptitudes, en tanto posibilidades de agencia en el propio útil (affordances).

El pensador distingue en su texto al cuerpo actual del habitual ${ }^{33}$. El cuerpo actual tiene presente, sin un esfuerzo deliberativo, los gestos de manejo y las técnicas de operatividad para llevar a cabo eficientemente su empleo. Por el otro lado, el cuerpo habitual requiere traer para sí en un acto rememorativo aquellas funciones latentes (habilidades incorporadas) que pueden activarse en su manejo. Recuérdese que este es el sentido del «movimiento virtual», aquel que requería de la producción de las condiciones necesarias para llevar a cabo la experiencia en un horizonte que no estaba dado sino que aparecía indeterminado. De este modo, tanto el cuerpo habitual como el movimiento virtual llevan al sujeto fuera del estado de absorto. Aunque, salir del estado de absorto no implica directamente transportarse a un nivel trascendental en el que se elaboraría la síntesis intelectual, que permitiría efectuar los arreglos oportunos para el movimiento concreto y actual. Por el contrario, este momento de adaptación y renovación del esquema corpóreo genera la restructuración en medio de la experiencia. De forma tal que, la síntesis perceptiva de la que habla Merleau-Ponty sostiene la unidad de lo externo y lo interno, aun en una instancia deliberativa ${ }^{34}$.

No obstante, se mantiene aún un problema. El hecho de hacer consciente para sí los procesos, capacidades o mecanismos involucrados en el uso de un determinado útil, conlleva un cambio de actitud en el sujeto. Pues, éste termina por objetivar la práctica y transformar al útil en objeto tético. El útil ya no es útil, pues se rompe el tejido de intenciones (la trama invisible de sentido) al explicitarlo. Al respecto, el filósofo se pregunta: «¿Cómo puedo percibir unos objetos como manejables, cuando no puedo manejarlos? [y responde] Es preciso que lo manejable haya dejado de ser lo que actualmente manejo, para devenir lo que puede manejarse, haya dejado de ser un manejable para mí y haya devenido como manejable en sí» ${ }^{35}$.

Bajo esta óptica, cuando el cuerpo deja de lado su función absorta en el sentido inmediato y toma las riendas la conciencia bajo la modalidad del cálculo, ¿se desvirtúa el sentido carnal que vivenciaba al útil? Si entendemos por «sentido carnal» al trato absorto en el marco del mundo vivido, la respuesta sería afirmativa. Por eso, «...cuanto más "ayoica” puede ser una actividad intencional compleja [...] o sea, cuanto menos reflexiva, menos memorativa, menos "articulativa” es, más clara

32 Merleau-Ponty, Php, p. 167/ p. 160.

33 Véase, Ibidem, pp. 97-98/ p. 101.

34 Véase, Ibidem, p. 166/p. 159.

35 Ibidem, p. 98/p. 101. 
e inequívocamente manifiesta la historia personal del yo que la acomete» ${ }^{36}$. Con esta frase habría que admitir que el hábito, en su versión latente, interrumpe la dinámica «original» del mundo vivido. Esto se debe a que al requerir explicitarse para activar la práctica de forma eficiente, rompe con la actitud natural que se daba en el acto. No obstante, al romper con la actitud natural y acceder a una actitud teórica ¿se visibiliza el espacio como extensión que excede la configuración del mundo vivido? Dejamos vigente nuestra pregunta inicial y nos sumergimos en la tercera clave del hábito para responder esto.

\section{LA CONTRIBUCIÓN DEL HÁBITO AL PROBLEMA DEL USO DEL ÚTIL}

Merleau-Ponty dice que: «el hábito expresa el poder que tenemos de dilatar nuestro ser-del-mundo o de cambiar la existencia anexándonos nuevos instrumentos. [...] Si el hábito no es ni un conocimiento ni un automatismo ¿qué será, pues? Se trata de un saber que está en las manos, que solamente se entrega al esfuerzo corpóreo y que no puede traducirse por una designación objetiva ${ }^{37}$. En este caso, contrario a lo que habíamos llegado al final del apartado anterior, por más que se plantee la necesidad de remontarnos a habilidades que no están presentes de momento o que se aprendan a medida que experimentamos los objetos, esto no generaría para el filósofo un quiebre del uso o del ser-a-la-mano de las habilidades implícitas en el uso de útiles. Pues, para dichas habilidades adquieren un carácter semiconsciente que funciona bajo la «intencionalidad operante» que sigue su cur$\mathrm{so}^{38}$. De esto se infiere que el útil no dejaría de ser útil si se perturbara su uso en el proceso. El punto está en que la inteligibilidad del acto pasa por la motricidad corporal y no por un recurso representativo propio de la «intencionalidad tética». No hay necesidad de apelar a representaciones que habiliten la práctica porque en el propio uso está dada, gracias a la motricidad involucrada, la significación del acto. En este sentido, las habilidades del sujeto son estrictamente corporales ${ }^{39}$, y no deben someterse a un instinto objetivante-como habíamos indicado en el apartado de la espacialidad.

Acorde con esto, habría otro tipo de atención — distinta a la objetivación- en la instancia elemental, que permitiría efectuar el ajuste de las condiciones sin violentar los hilos intencionales de la dinámica del mundo vivido. Ahora, para nosotros, esto no implica hablar de una imposibilidad de proyección fuera del campo fenomenal, al menos bajo la forma más laxa de una ampliación del mundo vivido. Justamente porque la idea del cuerpo habitual, es decir, las habilidades corporales incorporadas y latentes en el organismo son nuestra forma de conectarnos con las cosas que experimentamos. "El cuerpo habitual es algo que oficia de nexo y

36 SERRANO DE HaRo, o.c., p. 57.

37 Merleau-Ponty, Php, p. 168/p. 161. El subrayado es nuestro. Lo empleamos para acentuar la idea de un «yo puedo» en el cuerpo, más que un «yo pienso». (Cf. Merleau-Ponty, Php, p. 154)

38 Véase, García, o.c., pp. 52-53.

39 Cf. Ibidem, p. 56. 
también de terreno ontológico común entre el sujeto y el objeto» ${ }^{40}$. Pues, de hecho, vimos que el cuerpo representa el cruce de lo interno y lo externo a través de la unidad que nos da el esquema corpóreo y que el hábito aclara. Esto es así, ya que por medio del hábito logramos comprendernos en la práctica a partir del tipo de gestualidad y estilo motriz que ejercemos. Merleau-Ponty concluye la cuestión al sostener que: «...el fenómeno del hábito nos invita a [retomar] nuestra noción de "comprender" y nuestra noción de cuerpo» ${ }^{41}$. Pues, la comprensión se da como encuentro entre nuestra intención perceptiva y su efectuación motriz, y el cuerpo es justamente el medio para ello.

Con el cuerpo el filósofo llega a la idea de que su movimiento no debe considerarse como la expresión carnal de un pensamiento. «El sujeto pensante debe fundarse en el sujeto encarnado ${ }^{42}$. Del mismo modo, el espacio corpóreo no alude a un espacio previamente representado. La motricidad del cuerpo está íntimamente relacionada con el espacio en el que se desarrolla y proyecta al sujeto hacia la cosa en cuestión. De este modo, las habilidades del cuerpo son el efecto de un proceso de aprendizaje que se da con la práctica y produce el sentido de la cosa a partir de la dinámica motriz que se despliega. De la misma manera, si la habilidad requiere transformarse para acoplarse al progreso de su objeto, esto se realiza retomando el curso de la experiencia al atender en conjunto al movimiento y el fondo en el que se emplaza. "Un movimiento se aprende cuando el cuerpo lo ha aprendido, eso es, cuando lo ha incorporado a su "mundo" y mover su cuerpo es apuntar, a través del mismo, hacia las cosas, es dejarle que responda a la solicitación que éstas ejercen en él sin representación ninguna. [...] Para poder mover nuestro cuerpo hacia el objeto, se precisa, primero, que el objeto exista para él, es preciso, pues, que nuestro cuerpo no pertenezca a la región del "en sí" " ${ }^{43}$. Acaso que a la inversa, gracias al aprendizaje del cuerpo, se despliegue la significación al exterior para una institución de la práctica más allá de la esfera del mundo vivido — si es que esto es posible.

\section{CONCLUSIÓN}

Con este artículo quisimos visibilizar una problemática que, para nosotros, no excede al planteo fenomenológico. De hecho, en los escritos mencionados de Husserl, Heidegger y Merleau-Ponty es la base para elaborar un abordaje diferente al posicionamiento dualista de la experiencia. A propósito, la concepción del mundo vivido no escapa a las contradicciones de su inmanencia, sino que busca la forma de encarar las complicaciones que aparecen en el pasaje de una actitud práctica a una teórica. Merleau-Ponty es, a nuestro entender, quien mejor atiende (de los tres autores) a las limitaciones del abordaje fenoménico.

40 Cf. Ibidem, p. 111.

41 Merleau-Ponty, Php, p. 169/p. 162. Entre corchetes modificamos la traducción empleada del original «remanier».

42 Ibidem, p. 225/p. 210.

$43 \quad$ Ibidem, p. 161/p. 156. 
Particularmente, un análisis de la génesis de la agencia técnica, entendida como una modalidad de la praxis en el mundo, nos permite explorar esta problemática. Pues, al interpretar la dinámica que rige el trato con el útil y el modo de comprensión que en éste encontramos, se pone en acto la necesidad de un reajuste del sujeto frente a las condiciones que enturbian o reclaman una transformación del modo inmanente como se lleva a cabo la actividad.

En este sentido las claves del espacio, el cuerpo y el hábito dan cuenta de ese término medio en la propia constitución del sujeto que habilita la posibilidad de transfigurar el entorno de acuerdo a su co-implicancia. De este modo, Merleau-Ponty encuentra la manera para articular en su teoría la textura del mundo sin perder de vista el carácter híbrido que posee. Así pues, consideramos que no hay que perder de vista que el filósofo constantemente alude a esta doble constitución del sentido en el entramado de lo natural y cultural, atendiendo de este modo a la esencia misma del sujeto. Para ello, lleva su descripción a una dimensión ontológica en la cual la praxis aporta el «entre» en donde se emplaza la experiencia.

\section{BiBLIOGRAFÍA}

Battán Horenstein, A. (2004). Hacia una fenomenología de la corporeidad. M. Merleau-Ponty y el problema del dualismo. Córdoba: Universitas. Editorial Científica Universitaria.

Buhrman, T., Di Paolo, E. (2015). «The sense of agency. A phenomenological consequence of enacting sensorimotor schemes» en Phenomenology and The Cognitive Sciences Journal, noviembre, DOI 10.1007/s11097-015-9446-7.

Flores Hernández, . (2012). «Ideas para una fenomenología del cuerpo vivido» en Rabanaque, Luis (Ed.) Afectividad, razón y experiencia. Buenos Aires: Biblos, pp. 105-118.

García, E. (2012). Maurice Merleau-Ponty. Filosofía, corporalidad y percepción. Buenos Aires: Editorial Rhesis.

Husserl, E. (1952). Ideen zu einen reinen Phänomenologie und phänomenologischen Philosophie. Zweites Buch: Phänomenologische Untersuchungen zur Konstitution. The Hague: Martinus Nijhoff. [Trad. Ideas relativas a una fenomenología pura y una filosofía fenomenológica. Libro II: Investigaciones fenomenológicas sobre la constitución, FCE, México, 2005.]

Menéndez, R. (2015) «La indiscreta presencia de sí: La génesis de las habitualidades y el problema de la reflexión en Husserl» en Investigaciones Fenomenológicas, vol. Monográfico 5, pp. 193-218.

Merleau-Ponty, M. (1945). Phénoménologie de la perception. Paris: Gallimard. [Trad. Fenomenología de la percepción, Planeta-Agostini, Barcelona, 1993.]

Liberati, N. (2014). «Leib and Technologies: Relations and co-Fundation» en Investigaciones fenomenológicas, $\mathrm{N}^{\circ} 11$, pp. 165-184.

Pietersma, H. (2000). Phenomenological epistemology. New York-Oxford: Oxford University Press.

Rabanaque, L. (2012). «Afectividad, encarnación, razón» en Rabanaque, Luis (Ed.) Afectividad, razón y experiencia. Buenos Aires: Biblos, pp. 93-104.

Ramos González, J. A. (2014). «Lo avistado por Merleau-Ponty de la mano de Husserl: mundo, percepción y carne» en Éndoxa. Series filosóficas, N 34, pp. 209-230.

San Martín, J. (2010). «El contenido del cuerpo» en Investigaciones fenomenológicas, vol. Monográfico 2: Cuerpo y alteridad, pp. 169-187.

Serrano de Haro, A. (2007). La precisión del cuerpo. Análisis filosófico de la puntería. Madrid: Trotta. 
Toadvine, Te., Embree, L. (ed.). (2002). Merleau-Ponty's Reading of Husserl. Dordrecht-Boston-London: Springer Science + Business Media.

Verbeek, P. P. (2005). What Things do: philosophical reflections on Technology, Agency and Desing, trad. Robert P. Crease. Pennsylvania: Pennsylvania State University Press.

Universidad Nacional de Mar del Plata

Leticia Basso Monteverde

Facultades de Psicología y Humanidades

Consejo Nacional de Investigaciones Científicas y Técnicas.

leticiabassomonteverde@gmail.com

[Artículo aprobado para publicación en diciembre de 2016] $]^{44}$

${ }^{44}$ Revisado en julio de 2020. 